\title{
Die Druckerei der Propaganda Fide im Dienste der Glaubensverbreitung (II)
}

\author{
von Willi Henkel
}

Die Polyglotta von 1700 bis zur Zeit der Französischen Revolution (1789)

\section{Die ersten drei Jabrzehnte des 18. Jabrhunderts}

$\mathrm{Zu}$ Beginn des 18. Jahrhunderts standen wieder einmal finanzielle Probleme im Vordergrund. Kardinal Francesco Barberini bemühte sich deshalb um einen genauen Uberblick über die Ausgaben der Druckerei ${ }^{79}$. Um eine Vergleichsmöglichkeit zu haben, ordnete er 1716 an, die Kosten für die verflossenen 30 Jahre aufzustellen. Er ernannte ferner Francesco Apolonij zum Buchhalter und hoffte damit eine bessere Kontrolle der Finanzen zu erreichen.

Fast alle Entscheidungen der kanonischen Visitation von 1730 bis 1733 befaßten sich mit der wirtschaftlichen Lage ${ }^{80}$. So wurde auch das Heilige Offizium gebeten, die griechischen Bücher und andere Werke, die es bestellt hatte, selbst zu bezahlen. Es wurde ferner angeordnet, daß die Kardinäle nur noch ungebundene Exemplare von Büchern erhalten sollten. Der Rektor des Kollegs erhielt für die Ausgaben der Drukkerei nicht mehr 600 Scudi wie bisher, sondern nur noch 400 Scudi für ein Jahr. Die Kardinäle, welche die Visitation durchführten, verlangten einen Bericht darüber, ob die Bücher für die Kongregation oder für einzelne Persönlichkeiten gedruckt wurden. Wenn es sich um Privatpersonen handelte, sollte fortan die Erlaubnis des Papstes eingeholt werden. Schließlich wurde der Superintendent Nicolò Antonelli beauftragt, mit Buchhandlungen in Italien und im Ausland Kontakte aufzunehmen, um den Verkauf der Bücher zu erleichtern. Man stellte sodann eine Liste von Namen für eine Partikularkongregation zusammen, die dem Papst bei passender Gelegenheit Bericht erstatten sollte. Alle Entscheidungen der Visitation wurden am 18. Juni 1733 von Klemens XII. approbiert ${ }^{81}$.

Wenn man um diese Zeit von großen Ausgaben sprach, so kamen diese sicher nicht von den Löhnen der Angestellten, denn ihre Zahl war relativ gering. Es waren: Francesco Maria Acsamitek, der Sohn des früher erwähnten Zacharias Domenicus Acsamitek, Francesco Rossi, Giuseppe Collini, der Buchhalter Francesco Apolonij und ein Laufbursche ${ }^{82}$. Der sehr fähige Setzer Johann Jakob Komarek, der 11 Jahre für die Polyglotta gearbeitet hatte, eröffnete schließlich eine eigene Druckerei, nur wenige Meter von der Propaganda Fide entfernt, nachdem er bei der Polyglotta keine feste Anstellung erhalten konnte ${ }^{83}$.

Besondere Erwähnung verdient das vierbändige Werk „Bibliotheca Orientalis“ (1719-1728) von dem gelehrten Maroniten Joseph Simon Assemani, der Scriptor und Präfekt der Vatikanischen Bibliothek gewesen ist. Darin veröffentlichte er zahlreiche syrische Manuskripte, die aus Ägypten stammten und in der Vatikanbibliothek aufbewahrt werden ${ }^{84}$.

Dr. Willi Henkel OMI ist Direktor der Bibliothek der vatikanischen Kongregation für die Evangelisation der Völker (Propaganda) in Rom. Er ist Mitherausgeber der jährlich erscheinenden „Bibliographia Missionaria“. 


\section{Ein neuer Aufschwung}

Der Nachfolger von F. Nazzari als Superintendent der Polyglotta war Agostino Maria Taja ${ }^{85}$, der wegen seiner schlechten Gesundheit schon zwei Jahre später (1716) den Assistenten Simeoni erhielt ${ }^{86}$. Simeoni übernahm die Leitung der Druckerei im Jahre 1725, die er bis zu seinem Tode 1730 innehatte. Ein neuer Aufschwung bahnte sich unter seinem Nachfolger Nicold Antonelli an, der während des Konklaves von 1730 zum provisorischen Superintendenten ernannt und dann später von der Plenarversammlung der Kongregation bestätigt wurde ${ }^{87}$. Antonelli war ein Dichter und zugleich ein sehr praktischer Mann, der auf eine gute Ordnung achtete. Er sorgte für gutes Papier zum Drucken und schaffte eingerissene Mißbräuche ab. Die Lettern wurden wieder besser geordnet und auch nach Möglichkeit gebraucht ${ }^{88}$. Das war in den vergangenen Jahren häufig versäumt worden.

Antonelli verstand es auch, eine enge Zusammenarbeit mit dem Präfekten der Polyglotta, Kardinal Gentili, dem Sekretär der Kongregation, N. Lecari, und dem Rektor des Kollegs herzustellen. Im Jahre 1749 beschloß man außer den informellen Begegnungen, sich einmal im Jahr zu treffen, um die etwaigen Probleme eingehender zu diskutieren und auf längere Sicht im voraus zu planen ${ }^{89}$.

Der Superintendent entschied zusammen mit dem Sekretär der Kongregation und dem Rektor des Kollegs, welche Bücher für die Missionen nützlich waren und in der Polyglotta gedruckt werden sollten. Diesen gab man dann vor allen anderen Veröffentlichungen den Vorrang. So verwirklichte man die Vorschriften der Visitation von 1730 bis 1733 .

Auch nach seiner Ernennung zum Sekretär der Kongregation leitete er noch zwei Jahre lang die Druckerei. Als er dann erkannte, daß er der Polyglotta nicht jene Aufmerksamkeit schenken konnte, die eine gute Leitung erfordert, bat er im Jahre 1758 die Kardinäle der Kongregation, ihn von diesem Amt zu befreien und schlug Costantino Ruggieri als Nachfolger vor ${ }^{90}$.

\section{Der Höbepunkt der Polyglotta unter Ruggieri und Amaduzzi}

Obwohl Costantino Ruggieri nur fünf Jahre Superintendent der Druckerei gewesen ist, gehört er zweifellos zu den fähigsten Leitern, die sie je gehabt hat. Sein Nachfolger Amaduzzi rühmt die neuen Impulse, die er der Polyglotta gab und die ihr großes Ansehen verschafften ${ }^{91}$.

Eine Notiz, die von Ruggieri selbst stammt, zeigt uns den Eifer, mit dem er an die Arbeit ging. Sie spricht von den neuen Lettern, die in den ersten fünf Monaten seiner Leitung angeschafft wurden ${ }^{92}$. Darunter befinden sich armenische, arabische, chaldäische, hebräische, syrische und lateinische Typen, die erworben wurden. Aus der Gießerei von Urbino kamen weitere Lettern, ebenso auch von Kardinal Barberini. Vor allem der Bestand an syrischen Typen war sehr reichhaltig, so daß sich keine andere Druckerei mit der Polyglotta messen konnte. Neue chaldäische und armenische Lettern wurden für den Druck von Meßbiichern verwandt. Ruggieri versuchte auch, eine Anzahl armenischer Lettern von dem Kloster St. Lazzaro und über einen armenischen Priester in Holland zu bekommen. Weniger erfolgreich war er beim Beschaffen von arabischen Lettern, obwohl er auch diese von vier auf sechs Sätze erhöhte. Hebräische Typen kamen aus Paris. Schließlich gelang es Ruggieri, lateinische, armenische, arabische und syrische Lettern aus der vatikanischen Druckerei zu 
erhalten, die von den berühmten französischen Herstellern Garamond und Le Bé angefertigt worden waren und nicht mehr gebraucht wurden ${ }^{23}$.

Die Polyglotta besaß damals 27 verschiedene Arten von Typen. Aber auch diese große Anzahl genügte Ruggieri nicht. Bei passender Gelegenheit erinnerte er den Präfekten der Polyglotta daran, daß man neue Lettern anschaffen sollte. Diese Bitte trug Ruggieri sogar Papst Klemens XIII. vor ${ }^{94}$.

Ruggieri plante schließlich Bücher in malabarisch, Tamil und Telugu zu drucken. Die Anregung dazu kam durch den Besuch des protestantischen Missionars Benjamin Schultze in Rom, der zahlreiche Bücher in diesen Sprachen gedruckt hatte ${ }^{95}$.

Amaduzzi erwähnt eigens das Sachverständnis, mit dem Ruggieri neue Lettern anschaffte. Aber auch die alten ließ er nicht ungenutzt in den Kästen liegen, sondern reinigte sie und ersetzte sie, wenn es notwendig war. Um eine bessere Ordnung zu ermöglichen, schaffte er neue Schränke an.

Die eifrige Tätigkeit Ruggieris wurde von den Vorgesetzten der Kongregation anerkannt. Auf der Sitzung vom 14. April 1760 beschlossen die Kardinäle Spinelli als Präfekt der Kongregation, Tamburini als Präfekt der Polyglotta und Colonna Sciarra als Präfekt der Ơkonomie, Papst Klemens XIII. zu bitten, das jährliche Budget von 400 Scudi auf 1.200 Scudi zu erhöhen. Sie begründeten ihren Antrag mit der starken Nachfrage, vor allem von den orientalischen Kirchen nach Büchern in ihren Landessprachen. Wenn Rom nicht bereit oder imstande sei, diese Bitte zu erfüllen, würden sich diese Kirchen an die von Rom getrennten Gemeinschaften wenden. Die drei genannten Kardinäle wiesen auch auf das gute finanzielle Gleichgewicht der Kongregation hin, das ein herabgesetztes Budget nicht mehr länger erforderte. So könne man die Polyglotta in einer angemessenen Weise finanzieren, wie das von Anfang an geplant gewesen sei. Papst Klemens XIII. gab seine $\mathrm{Zu}$ stimmung für diesen Antrag ${ }^{06}$. So war es möglich, den Angestellten bessere Löhne auszuzahlen, und man konnte auch größeren Einsatz und bessere Qualität der Arbeit erwarten.

Im Jahre 1759 druckte die Polyglotta das Alphabetum Tibetanum, das von Bodoni verziert wurde. Der gelehrte Freund von Ruggieri, Augusto Antonio Giorgi, schrieb dazu die Einleitung, in der er die Sprache, Sitten und Gebräuche und die Religion der Tibeter erklärte ${ }^{97}$.

Unter Ruggieris Leitung erhielt die Druckerei auch einen neuen Sitz. 1759 wurde beschlossen, sie aus dem Erdgeschoß in den ersten und zweiten Stock auf der Westseite des Palastes zu verlegen. Der Superintendent erhielt ein eigenes Büro. Mit diesen Räumen war er vollauf zufrieden, wie wir aus seinem Bericht an Kardinal Spinelli über die Druckerei erfahren.

Aufgrund des genannten Berichtes kann man Ruggieri mit Recht als den ersten Historiker der Druckerei bezeichnen ${ }^{90}$. Darin gibt uns Ruggieri einen ausgezeichneten Ubberblick über die Tätigkeit der Druckerei während der ersten 120 Jahre ihrer Existenz. Er spricht von ihren Erfolgen und auch von den zahlreichen Schwierigkeiten, die sie zu überwinden hatte. Darüber hinaus stellt Ruggieri ein Programm für einen neuen Aufschwung auf. Sehr viele von diesen Punkten konnte er selbst verwirklichen, so z. B. die neue Unterkunft, die Ordnung der Lettern, neue Pressen und neue Alphabete. Mit Recht können wir Ruggieri „Kurator" und „Restaurator “ der Polyglotta nennen, wie ihn sein bekannter Nachfolger Amaduzzi bezeichnet. 
1761 veröffentlichte Ruggieri einen Katalog der Bücher der Polyglotta. Wie schon frühere Kataloge der Druckerei fand auch dieser besondere Beachtung und wurde auch nachgedruckt $t^{100}$. Er legt Zeugnis ab von der vielseitigen Produktion der Polyglotta.

\section{Bodoni in der Polyglotta}

Nur einige Monate nach der Ernennung Ruggieris zum Direktor der Polyglotta begann der damals 18jährige Giovanni Battista Bodoni, der schon bald einer der berühmtesten italienischen Drucker werden sollte ${ }^{\mathbf{1 0 1}}$, seine Lehre in der Propagandadruckerei.

Bodoni begann zunächst mit den Aufgaben eines Lehrlings, der die rostigen Lettern reinigte, die schon längere Zeit nicht mehr gebraucht worden waren, und sorgte sodann auch für Ordnung und Sauberkeit. Später lernte er das Gießen von neuen Lettern. Holzschnitte anzufertigen, hatte er bereits zu Hause bei seinem Vater in Saluzzo gelernt. Ruggieri entdeckte schon bald die außergewöhnlichen Fähigkeiten seines Lehrlings und suchte ihn auch tatkräftig zu fördern. Er sah, wie es diesem Freude bereitete, schwierige Holzschnitte anzufertigen, und wie er ständig Fortschritte machte. Ruggieri riet ihm, orientalische Sprachen an der Sapienza-Universität zu erlernen, die sehr nützlich für seine spätere Arbeit als Setzer waren ${ }^{102}$. In der Ausführung seiner Arbeiten war Bodoni stets sorgfältig und genau. Man gestattete ihm sogar, seinen Namen und Geburtsort Saluzzo auf Titelbilder von einigen Büchern zu setzen. Das war für den jungen Drucker und Künstler eine besondere Ehre und zugleich auch ein Ansporn. Bodoni setzte und dekorierte verschiedene arabischkoptische liturgische Bücher, die in der Polyglotta herauskamen, so z. B. das Pontificale, das Rituale und die Teotokia, welche der Professor und Bischof Raphael Tuki verfaßt hatte ${ }^{103}$. Die Titelblätter zu diesen Werken waren nicht gesetzt worden, sondern es handelte sich um Holzschnitte von Bodoni. Bald war Bodoni berühmter als sein Meister Alessandro Giardoni.

1766 verließ Bodoni die Polyglotta mit der Absicht, nach England zu gehen, um dort sein Gluick zu suchen. Sein Leben lang bewahrte er eine große Dankbarkeit für alles, was er in der Polyglotta gelernt hatte. Er sagte später einmal: „Ich kann meine Dankbarkeit für alle Wohltaten, die ich dort empfangen habe, nicht besser zeigen, als dadurch, daß ich öffentlich davon spreche. "104 Auch während der folgenden Jahre stand Bodoni im Briefverkehr mit den Leitern der Polyglotta, mit Amaduzzi und Cancellieri ${ }^{105}$. 1807 schickte er 4 Arten von Typen (Silvio, Littura, Testino und Garamoncino) in einem Wert von 2.000 Zecchini. Der Präfekt der Kongregation, Kardinal Di Pietro, schrieb in seiner Antwort, daß die Kongregation stolz auf ihren früheren Lehrling sei, der solchen Ruhm erlangt habe, und sie beneide die Druckerei von Parma um diesen fähigen $\mathrm{Mann}^{108}$.

\section{Ruggieris Nachfolger}

Zum Nachfolger Ruggieris wurde Ubaldo Bicci ernannt, der die Polyglotta von 1763 bis 1769 leitete $^{107}$. In den Jahren 1765 und 1769 gab er einen Katalog heraus. Von verschiedenen Seiten wurden auch Klagen laut, die Kongregation füge durch das Drucken von Büchern anderen Drudkereien Schaden $\mathrm{zu}^{108}$. Gerade das hatte man in der Vergangenheit stets zu vermeiden gesucht. - Wie Kardinal Barberini zu Beginn des Jahrhunderts, so bemühte sich Kardinal G. Castelli, der gleichzeitig Präfekt 
der Kongregation und der Polyglotta war (1763-1780), einen Oberblick über die Ausgaben der Druckerei zu verschaffen ${ }^{109}$.

\section{Amaduzzi Leiter der Polyglotta}

Giovanni Cristofero Amaduzzi gelang es, die Fortschritte weiterzuführen, welche die Polyglotta vor allem unter Ruggieri erreicht hatte. Er wurde am 10. Februar 1770 von Papst Klemens XIV. zum Superintendenten ernannt und leitete die Druckerei 22 Jahre lang ${ }^{110}$. Amaduzzi war vorher Professor der orientalischen Sprachen an der Sapienza-Universität gewesen. Aber auch in weiteren Wissensgebieten, wie in der Archeologie, der Geschichte und im Kirchenrecht, hatte er viel beachtete Beiträge geleiste $^{111}$. Amaduzzi war mit Ruggieri durch eine enge Freundschaft verbunden gewesen und wurde nicht weniger von dem Sekretär der Kongregation, Stefano Borgia, einem Freund und Förderer der Wissenschaften, geschätzt.

Wie Ruggieri, so bemühte sich nun Amaduzzi, neue Lettern anzuschaffen. Einige von diesen wurden von dem Gießer Giuseppe Expelli angefertigt. Der Sekretär der Kongregation, Stefano Borgia, schenkte der Druckerei ein etruskisches Alphabet ${ }^{112}$. Mit der Hilfe von weiteren Gelehrten veröffentlichte Amaduzzi zahlreiche Alphabete. Er selbst schrieb etwa zehn Einleitungen zu Alphabeten, in denen er Sprache, Land und Leute, Sitten und Gebräuche und die Religion der betreffenden Völker kurz beschreibt. Unter den Alphabeten waren auch die biblischen Sprachen Hebräisch und Griechisch vertreten, afrikanische Sprachen (Athiopisch), Sprachen des Vorderen Orients, Persisch und Armenisch, und des Orients, Tibetanisch, Malabarisch und Brahmanisch (je eines aus Hindustan und Ava). Weitere indische Alphabete wurden von dem gelehrten Karmelitermissionar Paulinus a S. Bartolomeo verfaßt $\mathrm{f}^{113}$.

Amaduzzi sorgte auch für eine gut geordnete Buchhaltung der Druckerei und fertigte Inventarverzeichnisse an. Wie seine Vorgänger machte er Vorschläge für neue Bücher, die für die Missionen nützlich waren. Nach einer sorgfältigen Prüfung der Ausgaben setzte er im Einvernehmen mit dem Sekretär und dem Präfekten der Kongregation die Preise für die Bücher fest ${ }^{114}$. Während Amaduzzis Amtsführung gestattete man auch Interessenten, Bücher in der Polyglotta zu drucken, wenn sie eine angemessene Zahlung leisteten und wenn feststand, daß andere Druckereien dadurch keinen Schaden nahmen. Auch die Herausgabe eines römischen Breviers wurde damals geplant; ihre Ausführung wurde jedoch durch ein unheilvolles Feuer verhindert ${ }^{115}$.

Ruggieri und Amaduzzi war es gelungen, die Drudkerei der Propaganda Fide zu größten Leistungen und zu höchster Qualität zu befähigen. $\mathrm{Zu}$ dem hohen Ansehen der Polyglotta trugen außer Bodoni auch Alessio und Giuseppe Giardoni, Benedetto Francesi und Antonio Fulgoni bei.

\section{Die letzten Jabre vor der Französischen Revolution}

Auf Amaduzzi folgte der Titularbischof Simone De Magistris, der die Polyglotta von 1792 bis 1802 leitete $^{116}$. Der Präfekt der Kongregation (bis 1795) und Präfekt der Polyglotta (bis 1811), Kardinal Leonardo Antonelli, ermutigte den Superintendenten, den Druck der Alphabete fortzusetzen ${ }^{117}$. Als Proto diente Antonio Fulgoni, der aber auch gleichzeitig für die wirtschaftlichen Belange des Kollegs sorgte ${ }^{118}$. Mit dem Herannahen der Französischen Revolution begann auch für die Polyglotta eine äußerst schwierige Zeit. 


\section{Die Polyglotta zur Zeit der Französischen Revolution}

Am 24. April 1797 erhielt Kardinal Antonelli die Nachricht vom Staatssekretariat, der Papst habe der Polyglotta die Erlaubnis erteilt, einige Alphabete für eine Ausstellung der orientalischen Lettern nach Paris zu schicken. Die Anfrage war vom französischen Minister Cacault ausgegangen ${ }^{119}$. Ein Begleitbrief des Direktors der Imprimerie de la Republique, Duboyraverne, und des Kurators der Orientalischen Manuskripte der französischen Nationalbibliothek, L'Hoengle, war in seiner Aussage noch deutlicher. Die Bevollmächtigten der französischen Regierung in Rom waren beauftragt worden, die notwendigen Maßnahmen zu treffen, um jeweils ein Exemplar aller ausländischen Lettern der Polyglotta zu beschaffen und so die großartige Sammlung von Paris zu vervollständigen ${ }^{120}$. So wurden 179939 Kästen mit arabischen, armenischen, brahmanischen, chaldäischen, koptischen, hebräischen, georgischen, griechischen, irischen, illyrischen, indischen, malabarischen, persischen, ruthenischen, syrischen, deutschen und tibetischen Lettern zur Französischen Akademie transportiert. Weitere Kästen mit arabischen, griechischen, syrischen und lateinischen Typen und einige Pressen wurden nach Civitavecchia und Ancona und von dort nach Korfu und Agypten befördert ${ }^{121}$. Auf geschickte Weise gelang es Antonio Fulgoni, eine Anzahl Lettern vom französischen Bevollmächtigten zu kaufen, die er im Vatikan unterbrachte, wo sie zwei Jahre lang blieben ${ }^{122}$.

Nachdem sich die politische Lage verbessert hatte, wurde die Polyglotta auch wieder geöffnet. 1802 ernannte die Kongregation der Propaganda Fide Francesco Cancellieri zum Superintendenten ${ }^{128}$. Auch er war, wie zahlreiche Vorgänger, ein Mann von großer Gelehrsamkeit. Der damals 80jährige Verwalter Antonio Fulgoni erhielt 1807 Francesco Bourlié als Assistenten, der vorher als Schriftsetzer gearbeitet hatte ${ }^{124}$. Auch während diesen schwierigen Jahren setzten drei Schriftsetzer, zwei Männer an der Presse und ein Gießer, die Arbeit fort ${ }^{125} .1809$ wurde sogar ein Wettbewerb veranstaltet, um den fähigsten Setzer zu ermitteln. Schiedsrichter in dieser Angelegenheit waren der Agyptologe G. Zoega, Professor M. A. Lanci und der Geistliche De Rossi ${ }^{129}$.

Von neuem befand sich die Polyglotta 1810 in einer schwierigen Lage, als der Generalsekretär des französischen Innenministeriums eine weitere Sendung von Lettern verlangte. Cancellieri versuchte ihn zu überzeugen, daß seine Bemühungen nur einen geringen Ertrag bringen könnten, da die Druckerei von Paris schon im Besitz von Lettern in 250 Sprachen sei ${ }^{127}$. Der Minister beharrte jedoch auf seiner Forderung, wenigstens einige Duplikate zu schicken ${ }^{128}$. So wurden dieses Mal 1890 orientalische Lettern nach Paris geschickt ${ }^{129}$. Es gelang F. Bourlié, 8961 Lettern in 27 verschiedenen Sprachen zu retten, unter denen sich auch die Typen befanden, die Bodoni der Polyglotta geschenkt hatte. Sie wurden an einem sicheren Ort versteckt ${ }^{130}$.

Am 6. Juli 1812 wurde die Druckerei nochmals zwangsweise geschlossen. Diese Maßnahme sollte jedoch nicht lange dauern, denn die politische Lage änderte sich schon bald. Am 1. April 1814 konnte die Polyglotta wieder geöffnet werden ${ }^{131}$. Ein Jahr später (1815) erfahren wir, daß sich der Staatssekretär, Kardinal Consalvi, im Auftrage von Papst Pius VII. um die Zurücksendung der Lettern der Polyglotta aus Paris bemuihte. Msgr. Marino Marini wurde diese Aufgabe aufgetragen ${ }^{132}$. Die Erlaubnis dazu wurde in Paris erteilt. Es bleibt jedoch ungewiß, ob die Rückerstattung tatsächlich ausgeführt wurde, weil die Akten des Propagandaarchivs keinen Aufschluß darüber geben. 


\section{Ein neuer Anfang}

Zwei Jahre nach der Wiedereröffnung der Druckerei (1816) wurde diese wiederum in das Erdgeschoß verlegt. Der Plan für die neuen Räume war vom Architekten Nicoletti entworfen worden ${ }^{133}$. Damit wurde ein Beschluß ausgeführt, der bereits 1792 gefaßt worden war, nachdem man bemerkt hatte, daß die Pressen Schaden am Gebäude anrichteten. Der Grund für die späte Ausführung des Dekrets mag wohl die Französische Revolution gewesen sein. Die Druckerei hatte nun einen eigenen Eingang und störte nicht mehr den Tagesablauf des Kollegs.

1815 erhielt der Superintendent den Geistlichen Pietro Gambini als Assistenten, der jedoch beim Tode von Cancellieri im Jahre 1826 nicht sein Nachfolger wurde ${ }^{134}$. Als Grund wird sein machiavellistischer Charakter angegeben.

Von 1829 bis 1839 leitete Paul Cullen die Druckerei, der Professor für Hebräisch und für orientalische Sprachen am Propaganda-Kolleg war ${ }^{135}$. Unter seiner Leitung wurde die erste Sammlung von Dokumenten der Kongregation veröffentlicht, die den Titel „Bullarium Pontificium Sacrae Congregationis de Propaganda Fide“ trägt ${ }^{138}$.

In den zwanziger und dreißiger Jahren versuchten die Verwalter ihren Einfluß zu verstärken. Francesco Bourlié hatte sich große Verdienste während der französischen Besetzung Roms erworben und verstand es auch, sich am 9. Dezember 1821 eine schriftliche Anerkennung von Pius VII. zu verschaffen ${ }^{137}$. Von 1820 an wurde sein Sohn Leopold sein Gehilfe in der Ausübung seines Amtes als Verwalter, 1830 wurde er sein Nachfolger ${ }^{138}$. Die Familie Bourlié sprach gern von ihren erworbenen Rechten. 1834 erhielt Bourlié die Erlaubnis, auch Bücher für auswärtige Personen auf deren Kosten zu drucken ${ }^{139}$. Um den Verkauf der Bücher zu erleichtern, gewährte der Sekretär der Kongregation, A. Mai, den Verwaltern einen Rabatt von $10 \%$. Wenn sie die Bücher an Buchhändler verkauften, betrug er $5 \% 0^{140}$.

\section{Modernisierung der Druckerei unter De Luca}

Der Nachfolger von Cullen, Antonio Saverio De Luca, bemühte sich, die neuen Drudkverfahren, die damals aufkamen, in der Polyglotta einzuführen ${ }^{141}$. Er beauftragte den Pariser Proto, August d'Urtubié, im Jahre 1841, die Druckerei neu zu organisieren und sich die französischen Fortschritte zunutze zu machen ${ }^{142}$. Dazu verwandte er das bereits vorhandene Material und ließ auch neue Lettern gießen. Eine Anzahl von Typen kamen aus dem Vatikan; ferner wurden $87 \mathrm{Kästen} \mathrm{mit} \mathrm{Lettern}$ von der Pariser Firma Didot erworben ${ }^{143}$. Es wurden auch neue gußeiserne Pressen und eine hydraulische Presse in Paris bestell $t^{144}$. Neue Möbel für die Lettern sollten zu einer besseren Ordnung verhelfen. 1842 wurden auch die Bestimmungen für die Angestellten neu formuliert ${ }^{145}$. Als De Luca im Jahre 1845 zum Bischof von Aversa ernannt wurde, war die Druckerei der Propaganda Fide wieder auf einen solchen Stand gebracht, daß sie sich mit anderen europäischen Druckereien messen konnte.

Von den Kardinälen, die in jenen Jahren für die Polyglotta verantwortlich waren, verdient Mauro Cappellari besondere Erwähnung, der von 1826 bis 1831 Präfekt der Propaganda Fide war. Auch als Papst (Gregor XVI.) bekundete er der Polyglotta sein Interesse für ihre Tätigkeit durch einen Besuch im Jahre 1842. Bei dieser Gelegenheit wurde ihm ein Band überreicht, der in Lettern verschiedener Sprachen der Polyglotta geschrieben war; es waren 27 europäische, 22 asiatische, 3 afrikanische und 3 amerikanische Sprachen darin vertreten ${ }^{146}$. 
Nachdem A. Bourlié ein Jahr lang unter der Leitung des französischen Proto d'Urtubié gearbeitet hatte, bewarb er sich um diesen Posten, den er auch im Jahre 1843 erhielt $^{147}$. Bourlié unterschätzte die Rolle des Superintendenten. Er betrachtete die Tätigkeit der Druckerei aus der Perspektive des Proto, der zweifellos eine wichtige Aufgabe im Hinblick auf den technischen Ablauf der Arbeiten der Druckerei erfüllt. Er sollte auf gute Ausführung der verschiedenen Dienstleistungen achten und auch für eine gute Ordnung der Lettern sorgen. Gerade das war der schwache Punkt, den die Visitation von 1856 aufdeckte.

\section{Die Visitation von 1856}

Obwohl Bourlié im Jahre 1853 die einzigartige Chance bekam, zwei Monate lang in Paris moderne Druckmethoden kenenzulernen, zögerte er, diese seinen Vorgesetzten vorzuschlagen, weil er befürchtete, daß in diesem Falle eine Reihe von Angestellten entlassen werden müßten ${ }^{148}$. Er verfolgte eine andere Politik, die sich darin äußerte, daß er in dem kurzen Zeitraum von drei Jahren 12 Personen anstellte und damit die Zahl der Angestellten im Jahre 1852 verdoppelte ${ }^{\mathbf{1 4 9}}$. Er glaubte immer noch an die traditionellen Methoden der Handarbeit und war den neuen Maschinen gegenüber skeptisch. Dem Anwachsen des Personals entsprach jedoch nicht die Organisierung der Arbeit. Obwohl die letzten Arbeitsbestimmungen erst 1842 aufgestellt worden waren, war ein deutlicher Mangel an geordneter Zusammenarbeit zu spüren ${ }^{150}$. Darüber sollte ein eigens bestellter Visitator das Urteil abgeben.

Mit dieser Aufgabe beauftragte die Kongregation der Propaganda Fide Benigno Scalabrini, der am 21. Juli von dem Sekretär der Kongregation Alessandro Barnabò dem Personal der Polyglotta vorgestellt wurde. Von Anfang an gaben einige Angestellte nur zögernd Auskunft auf die Fragen des Visitators; sie wichen aus, indem sie sagten, sie seien nicht daran gewohnt, die Arbeit auf eine bestimmte Art und Weise $\mathrm{zu}$ verrichten ${ }^{151}$.

Scalabrini gibt uns ein detailliertes Bild über den Zustand der Polyglotta und macht schließlich eine Reihe von Vorschlägen für Verbesserungen ${ }^{152}$. Zuallererst nennt er den Mangel an Ordnung bei der Buchführung und das Fehlen von Listen über die Bücher, die ins Ausland geschickt worden waren. Es scheint, daß Bourlié keine Abrechnungen für das Papier verlangte. Die Arbeiter folgten keinem bestimmten Arbeitsplan. Eine Anzahl von Lettern wurden vermißt, wieder andere befanden sich in Unordnung. Ein Teil war abgenutzt und diente nicht mehr für den weiteren Gebrauch. Die Buchhandlung registrierte nur die verschickten Bücher, während die Anzahl der erhaltenen Bücher fehlte. Der Lagerraum befand sich in einer so großen Unordnung, daß der verantwortliche Angestellte Angelo Grilli entlassen werden mußte.

Scalabrini machte insgesamt 27 Vorschläge, die er für eine geordnete Arbeit als notwendig erachtete ${ }^{153}$. Er wies auf das Einholen der Erlaubnis für den Druck neuer Bücher hin. Diese war auch notwendig für den Abschluß neuer Verträge. Er schlug vor, daß einmal im Monat ein Rechenschaftsbericht abgefaßt werden sollte. Außerdem hielt er einen jährlichen Überblick für notwendig.

Der Visitator machte ferner den Vorschlag, einen besonderen Eingang für jenen Teil der Druckerei zu schaffen, in dem die Bücher gedruckt wurden, die nicht für die Offentlichkeit bestimmt waren. Die Autoren sollten sich nicht mehr mit den Setzern 
direkt in Verbindung setzen, sondern mit dem Proto verhandeln, der die verschiedenen Etappen der Ausführung beaufsichtigt.

Schließlich schlug Scalabrini vor, festgesetzte Löhne zu bezahlen. Die Arbeitszeit sollte 10 Stunden am Tag betragen.

Am 22. April überreichte Scalabrini Kardinal Pietro Marini den Visitationsbericht ${ }^{154}$. Bourlié versuchte sich, so gut er konnte, zu verteidigen. Er gab auch zu, Fehler gemacht $\mathrm{zu}$ haben ${ }^{155}$. Als Hauptgrund führte er an, die Angestellten hätten während seines Aufenthaltes in Paris das Vertrauen $\mathrm{zu}$ ihm verloren ${ }^{156}$. Schließlich beschuldigten die Arbeiter den Proto der Nachlässigkeit in der Erfüllung seiner Aufgabe ${ }^{157}$. Unter diesen Umständen konnte er sie nicht fortführen, und er wurde am 9. Juni 1856 entlassen.

Barnaba Tortolini, der bisher Professor am Propaganda-Fide-Kolleg gewesen war, wurde zum neuen Superintendenten ernannt ${ }^{158}$. Die Verwaltung des Lagerraums wurde Paolo Rosa übertragen, der dem Superintendenten nicht länger unterstand, sondern selbständig war.

Tortolini wurde jedoch in einen Prozeß verwickelt, der einen großen Teil seiner Aufmerksamkeit erforderte ${ }^{159}$. Er sah sich gezwungen, im Jahre 1863, um Entlassung aus seinem Amt zu bitten ${ }^{160}$.

Wieder einmal war die Kongregation der Propaganda Fide der Meinung, die Ausgaben für die Druckerei seien im Gesamtbudget zu hoch. Deshalb bemühte man sich um neue Lösungen.

\section{Pietro Marietti Verwalter und gleichzeitiger Eigentümer der Polyglotta}

Die größte Anzahl der Kardinäle der Kongregation stimmte dem Vorschlag zu, die Druckerei einem bekannten Verleger anzuvertrauen. Der italienische Druckereibesitzer Pietro Marietti erklärte sich bereit, die Verwaltung der Polyglotta zu übernehmen. Am 22. August 1864 beauftragte die Generalkongregation eine Kommission mit dem Entwurf eines Vertrages, der dann auch am 16. Januar 1865 von der Generalversammlung angenommen wurde, nachdem einige Anderungen eingearbeitet worden waren ${ }^{161}$.

Kurz darauf, nachdem der Vertrag unterzeichnet worden war, informierte Pietro Marietti alle katholischen Buchhandlungen und Verleger, daß eine Gesellschaft der Polyglotta gebildet worden sei und er mit der Verwaltung der Druckerei beauftragt sei $^{162}$. Papst Pius IX. richtete ein Schreiben an den neuen Verwalter der Polyglotta, in dem er von der Bedeutung der kirchlichen Presse sprach und Marietti in seinem Unternehmen ermutigte ${ }^{163}$. Darüberhinaus stattete der Papst der Druckerei am 23. Mai 1866 einen Besuch ab, den man als Ausdruck seines persönlichen Interesses werten darf. Auch bei dieser Gelegenheit wurde dem Papst ein Band überreicht, der die Vielfalt der Lettern der Druckerei deutlich veranschaulicht. Darin wurde der 7. Vers des vierten Kapitels vom Buch Deuteronomium in 250 Sprachen dargebo$\operatorname{ten}^{104}$.

Nachdem Marietti die Druckerei übernommen hatte, wurde ein weiterer Raum für die Maschinen zur Verfügung gestellt. Durch den Kauf einer neuen Druckmaschine von der Firma König und Bauer aus Oberzell bei Würzburg wurde der Druckablauf wesentlich beschleunigt ${ }^{165}$. Es wurde auch eine moderne Falzmaschine angeschafft, welche die erste dieser Art in Italien war. Beide Maschinen wurden mit Dampf 
angetrieben. Eine weitere Maschine druckte in zwei Farben. Die Druckerei bediente sich auch der neuen Methode der Stereotypie und stellte Gravierungen her nach der Technik, die Giuseppe Gozza entwickelt hatte und die eine sehr große Präzision ermöglichte. Die Aufgabe des Proto erfüllte C. Griffin, der 14 Arbeiter unter sich hatte.

Von den Veröffentlichungen der damaligen Zeit verdient der Vorläufer der heutigen "Acta Apostolicae Sedis“ besondere Erwähnung" ${ }^{166}$. Der berühmte protestantische Exeget Constantin von Tischendorf schlug Papst Pius IX. vor, den Codex Vaticanus zu veröffentlichen. Der Papst beauftragte den Barnabiten Carlo Vercellone mit der Herausgabe. Tischendorf war auch beim Beschaffen von geeigneten Lettern behilflich. Pius IX. unterstützte diese kostspielige Arbeit durch den Kauf von 12 Exemplaren ${ }^{187}$.

Schon nach sechs Jahren der Verwaltung beabsichtigte Marietti den Vertrag zu lösen ${ }^{188}$. Als Gründe führt er die wirtschaftlichen Schwierigkeiten der damaligen Zeit an. Er beklagt auch das mangelnde Interesse für Bücher, welche die Mission zum Gegenstand haben. Auch Nachdrucke verursachten oft große Schwierigkeiten. Außerdem war Marietti in einen Prozeß mit Pallotini ${ }^{109}$ und in einen weiteren mit Angelo Lucini verwickelt ${ }^{170}$. Deshalb zog sich Marietti am 14. Juni 1872 aus der Verwaltung zurück und schlug als seinen Nachfolger Frederico Melandri vor, der bis zu diesem Zeitpunkt die Druckerei von Marietti in Turin geleitet hatte ${ }^{171}$. Als Melandri die Verwaltung der Polyglotta am 11. Juli 1872 übernahm, war die Zahl der Angestellten auf sechs gesunken ${ }^{172}$.

\section{Die letzten Direktoren der Polyglotta}

Auch Melandri gelang es nicht, die wirtschaftliche Lage der Druckerei wesentlich zu verbessern. Schon nach sieben Jahren seiner Verwaltung schrieb er einen Bericht, in dem er den langsamen und kaum merkbaren finanziellen Fortschritt zu analysieren versucht ${ }^{173}$. Daraus ergibt sich, daß die Bücher der Polyglotta nicht zu jenem Preis verkauft wurden, der eine günstige Gewinnspanne ergab. Er weist auch darauf hin, daß die wirtschaftliche Lage für die Verlage nicht ermutigend sei. Dennoch unterbreitet er verschiedene Vorschläge, um einen höheren Gewinn zu erzielen. So rät er, größere Werke wie z. B. die lateinischen und die griechischen Väter zu drudken. In der folgenden Zeit begann er den Druck der Werke von Thomas von Aquin. Für ihre Veröffentlichung wurde eine moderne Maschine von König und Bauer aus Oberzell angeschafft ${ }^{174}$. Die Druckerei wurde nochmals erweitert, und neue Lettern wurden aus Wien und aus Genf erworben ${ }^{175}$.

Ein weiterer Bericht von Melandri, der zehn Jahre später verfaßt ist, spricht von 34 Angestellten der Polyglotta, an deren Spitze B. B. Jacobini als Proto stand ${ }^{176}$. Melandri äußert darin seine Zufriedenheit mit den letzten Errungenschaften der Druckerei. Er ist auch optimistisch in Bezug auf das wirtschaftliche Wachstum des Unternehmens, wenn man fortfahre, größere Werke zu veröffentlichen. Er kritisiert aber die bisherige Produktion der Polyglotta, die zwar außergewöhnlich reich an Werken in fremden Sprachen sei, aber nur wenige Bücher größeren Umfangs gedrudkt habe. Doch schon bald danach begann man mit dem Druck des bedeutenden Werkes "Juris Pontificii de Propaganda Fide“, das von Raffaele De Martinis bearbeitet wurde. Der erste Teil veröffentlicht Akten des Papstes und der römischen Kurie in sechs stattlichen Bänden, und der zweite einbändige Teil enthält Dekrete der Propaganda Fide ${ }^{177}$. 
Der erwartete wirtschaftliche Aufschwung kam jedoch nicht. So äußerte der Präfekt der Okonomie, Kardinal Gaetano De Ruggiero, ernsthafte Bedenken über die Rentabilität der Druckerei. Aus diesem Grund wurde im Jahre 1890 eine Kommission eingesetzt, welche die wirtschaftliche Lage der Polyglotta studieren und neue Möglichkeiten erwägen sollte. Wiederum wies man darauf hin, daß man nur solche Bücher drucken dürfe, die mit den Zielen der Kongregation der Propaganda Fide übereinstimmten ${ }^{178}$. So ist es verständlich, daß Melandri um seine Entlassung bat. An seine Stelle trat Pietro Gatti, der seit 1884 sein Assistent gewesen war.

Die vorgeschlagene Verlegung der Druckerei wurde zunächst noch nicht ausgeführt, weil man die Möglichkeiten eines Vertrages mit einem anderen führenden Verlag ausfindig machen wollte. Man erkundigte sich bei Desclee, Pustet, Vives und Mareggiani, fand jedoch kein Interesse an einer Ubernahme der Druckerei.

So wurde die Druckerei im Jahre 1891 in den Palast Mignanelli verlegt, und Raffaele Mareggiani überwachte den Umzug.

Die Kongregation der Propaganda Fide äußerte den Wunsch, daß die Verwaltung des Direktors nicht mehr selbständig sein solle. Gatti bemühte sich auch, angemessene Preise für die Bücher zu errechnen ${ }^{179}$. Nicola Gaetano wurde zum Proto der 48 Angestellten bestimmt. Ihre Zahl wurde jedoch im Jahre 1900 auf 44 und 1906 auf 43 reduziert. Es ist verständlich, daß keiner der Arbeiter seinen Platz verlieren wollte. Schon im Jahre 1882 hatten sie aus diesem Grunde nicht an einem allgemeinen Streik teilgenommen, bei dem es sich um die Kranken- und Unfallversicherung handelte. Sie waren der Meinung, daß genügend für sie gesorgt sei.

Pietro Gatti bemühte sich tatsächlich um einen Fonds für Notfälle. Ahnliche Uberlegungen stellte auch Kardinal A. Ciasca an. Ebenso versuchte Gatti einen allgemeinen wirtschaftlichen Fortschritt der Druckerei zu erreichen.

Noch einmal versuchte die Kongregation der Propaganda Fide, die Polyglotta in Pacht zu geben. Sie führte im Jahre 1907 Verhandlungen mit dem Direktor der Tipografia Vaticana G. Pasquale Scotti, der die Druckerei zwei Jahre lang provisorisch leitete und zunächst auch geneigt zu sein schien, das Angebot anzunehmen. Als Bedingung stellte er jedoch, daß mehrere Arbeiter entlassen werden sollten. Es scheint, daß die Bereitschaft, darauf einzugehen, nicht vorhanden war. So kamen auch diese Verhandlungen zu keinem positiven Ergebnis.

Eines der letzten Werke, das die Polyglotta veröffentlichte, waren die "Collectanea S. Congregationis de Propaganda Fide“, eine chronologisch geordnete Sammlung der wichtigsten Dokumente der Kongregation über die Missionen ${ }^{180}$.

\section{Rückblick}

Wenn man die fast dreihundertjährige Geschichte mit ihrem Auf und Ab überfliegt, fesseln vor allem jene Persönlichkeiten, die voll und ganz an die Aufgaben der Polyglotta glaubten und deshalb auch imstande waren, die häufigen finanziellen Schwierigkeiten zu überwinden. Die erste Stelle gebührt Ingoli, dessen Reden nicht nur diesem Ziele dienten, sondern darüber hinaus auch eine bleibende Bedeutung haben, weil sie grundsätzliche Aussagen über die Bedeutung der Pressearbeit für die Mission enthalten. Als Historiker der Polyglotta kommt sodann Ruggieri ein besonderer Platz zu. Sein Bericht gibt schließlich, vor allem wegen seiner Vergleiche mit den protestantischen Druckereien jener Zeit, interessante Einblicke in die Missionspresse des 18. Jahrhunderts. 
Die Polyglotta wurde wegen der Qualität ihrer Drucke und der Vielzahl ihrer Lettern in der ganzen Welt sehr hochgeschätzt. Wie bei ähnlichen Unternehmen, z. B. bei Regierungsdruckereien, zielten die Bemühungen der Propagandadruckerei auf Qualitätsarbeit ${ }^{181}$. Investierungen wurden nicht vom Standpunkt des Gewinnes aus betrachtet, sondern waren von der gesamten finanziellen Lage der Kongregation abhängig und veränderten sich in dem Maße, wie die verantwortlichen Leiter die Prioritäten für dieses Missionsmittel setzten. Mit der hereinbrechenden Industrialisierung waren zusätzliche Investitionen notwendig. Obwohl es nicht leicht war, mit den Erfordernissen der Zeit Schritt zu halten, wurden auch in dieser Zeit große Anstrengungen für die Modernisierung der Druckerei gemacht. Die allgemeine wirtschaftliche Lage in der zweiten Hälfte des 19. Jahrhunderts war jedoch wenig günstig für eine weitere Aufwärtsentwicklung. Schließlich gründeten mehrere Ordensgemeinschaften in der zweiten Hälfte des 19. Jahrhunderts Druckereien in den Missionsländern ${ }^{182}$.

Als die römische Kurie im Jahre 1908 neugeordnet wurde, beschloß man, die Drukkerei der Propaganda Fide mit der vatikanischen Druckerei zu vereinigen, die seit dieser Zeit „Tipografia Poliglotta Vaticana“ heiß $\mathbf{t}^{183}$.

Anmerkungen:

79. SC Stamperia vol. 1 f. 705 rv.

80. SC Stamperia vol. 2 f. 69r-70v; vgl. audh SC Visite e Collegi vol. 40, 1733, 171r bis 173r; 329r-370r. Das Ergebnis der Visitation wurde dem Personal der Polyglotta am 1. März 1733 mitgeteilt.

81. SC Stamperia vol. 2 f. $71 \mathrm{v}$.

82. Acta vol. 61,1691, f. 7r; vol. 62,1692 , f. 59v; vol. 67,1697 , f. 286 r.

83. Acta vol. 54, 1684, f. 35v; vol. 61, 1691, f. 7rv. J. Komarek wurde um 1650 in Königgrätz geboren. Er war ein Neffe von Z. D. Acsamitek. Seine Druckerei befand sich in der Via S. Vincenzo bei der Piazza Trevi. Er starb vor 1710. Uber ihn vgl. A. Tinto: Giovanni Giacomo Komarek tipografo a Roma nei secoli XVII-XVIII ed i suoi campionari di caratteri. In: „La Bibliofilia" 75 : Firenze 1973, 189-225.

84. J. S. Assemani 1687 in Tripolis (Libanon) geboren. 1766 wurde er Titularerzbischof von Tyrus. Er starb 1768 in Rom.

85. Acta vol. 84, 1714, f. 652r; vol. 86, 1714, f. 198 r.

86. Acta vol. 86,1714, f. $198 \mathrm{r}-199 \mathrm{v}$.

87. SC Stamperia vol. 3 f. 433r. Nicolò Maria Antonelli Graf von Pergola wurde am 8. Juli 1698 geboren. Er leitete die Polyglotta von 1730-1758. Im Jahre 1756 wurde er zum Sekretär der Kongregation der Propaganda Fide ernannt; 1758 Kardinal. Er starb am 25.9.1767. Seine Bibliographie findet sich in H. Hurter: Nomenclator

89. SC Stamperia vol. 2 f. $429 r-431$ v.

90. SC Stamperia vol. 2 f. 431 rv.

91. SC Stamperia vol. 3 f. 433v. - Seine Biographie schrieb G. C. Amaduzzi: Commentarius in vitam viri cl. Constantini Ruggerii. In: Nuova Raccolta Calogeriana di opuscoli scientifici e filologici T. XX, Venezia 1770, 54-104. - Seine Bibliographie findet sich in: Biografia Universale Antica e Moderna vol. 49, Venezia 1829, 359-360. - Ruggieri nahm sich das Leben. Amaduzzi spricht von seiner Krankheit und Melancholie. Der Tod seines Protektors Kard. Spinelli betrübte ihn tief.

92. SC Stamperia vol. 2 f. $148 \mathrm{r}-151 \mathrm{v}$.

93. SC Stamperia vol. 2 f. 15r-23r. G. C. Amaduzzi: Commentarius S. 71. Die Vatikanische Bibliothek beanspruchte später die Lettern, weil sie nur geliehen worden seien. Ruggieri hingegen spricht von einem Geschenk Klemens XIII.

94. G. C. Amaduzzi: Commentarius S. 75.

95. G. C. Amaduzzi: Commentarius S. 74.

96. SC Stamperia vol. 2 f. 233 r, $432 v$. 
97. Die verschiedenen Ausgaben finden sid in: R. Streit - J. Dindinger: Bibliotheca Missionum vol. VI, Nr. 573, 583, 693. - Die Lettern wurden von dem Kapuziner und Tibetmissionar Orazio della Penna entworfen und auf Kosten des Kardinals Belluga von Antonio Fantozzi gegossen. Vgl. A. Giorgi: Alphabetum Tibetanum, Roma 1772, $559-560$.

98. SC Stamperia vol. 2 f. 172 r-173r.

99. Der Bericht von Ruggieri wird erstmals in: "Communicatio Socialis" ab Heft 1:1976 in Fortsetzungen veröffentlicht.

100. M. Galeotti: Della Tipografia Poliglotta di Propaganda, Torino 1866, 76.

101. Vgl. S. Samek Ludovici: Giovan Battista Bodoni e la Propaganda Fide. In: „Accademie e Biblioteche d'Italia" 33: Roma 1965, Nr. 3 S. 1-17. G. Aliprandi: G. B. Bodoni e l'opera sua, Padova 1940.

102. Passerini: Memorie aneddote per servire un giorno alla vita del signor B. B. Bodoni tipografo di S. Maestà Cattolica e direttore del parmense Tipografeo. Stamperia Carmignani (Parma 1804) 9. S. Samek Ludovici: Giovan Battista Bodoni e la Propaganda Fide 4-5.

103. Vgl. S. Samek Ludovici: Giovan Battista Bodoni e la Propaganda Fide S. 6-7. Das Pontificale wurde 1762, das Rituale 1763 und die Theotokia 1764 veröffentlicht.

104. G. B. Bodoni: Pel solenne battesimo di S. A. S. Ludovico principe primogenito di Parma tenuto al sacro fonte da Sua Maestà Cristianissima e dalla Reale Principessa delle Asturie. Iscrizioni esotiche e caratteri novellamente incisi e fusi. R. Stamperia, Parma, 18 aprile 1774, S. 7.

105. Im Jahre 1791 veröffentlichte G. B. Bodoni den Brief: Iohannis Christoferi Amadutii Epistola Ad Iohannem Baptistam Bodonium Qua Emendetur Et Suppletur Commentarium de Anacreontis Genere Eiusque Bibliotheca.

106. SC Stamperia Misc. vol. 1 f. 137r. Bodonis Antwort befindet sich auf f. 157rv. Weitere Briefe wurden veröffentlicht in: G. B. Bodoni e la Propaganda Fide. Museo Bodoniano, Parma 1959.

107. SC Stamperia vol. 3 f. 433v.

108. SC Stamperia vol. 2 f. 580 .

109. SC Stamperia vol. 2 f. 1013 r. U. Bicci hinterließ bei seinem Tod beachtliche Schulden; vgl. CP vol. 137 f. $340 \mathrm{r}-344 \mathrm{r}$.

110. SC Stamperia vol. 2 f. 1025r. Seine Bibliographie befindet sich in: „Biografia Universale Antica e Moderna" vol. 1: Venezia 1822, 230.

111. Amaduzzi zog die Leitung der Polyglotta der ehrenvollen Ernennung zum Sekretär der Accademia Reale delle Scienze delle belle Arti di Napoli vor.

112. M. Galeotti: Della Tipografia Poliglotta di Propaganda, Torino 1866, 78.

113. Paulinus a S. Bartolomeo (Johann Philipp Vesdin) OCD wurde 1748 in Hof, NiederOsterreich geboren. 1768 OCD; 1774-1776 reiste er nach Indien, 1786-1789 Missionar in Malabar. 1790 Prof. am Missionsseminar S. Pancrazio, 1803 Studienpräfekt am Propaganda-Kolleg; starb 1806 in Rom.

114. CP vol. $137 \mathrm{f} .116 \mathrm{r}$.

115. SC Stamperia vol. 3 f. 392v.

116. Simone De Magistris wurde 1728 in Serra di Scopomene, Sizilien geboren. 1791 wurde er Titularbischof von Cirene. Er starb am 6. Oktober 1802. Seine Bibliographie befindet sich in: Enciclopedia Cattolica vol. IV, Sp. 1389-1390.

117. SC Stamperia vol. 3 f. $426 r$.

118. SC Stamperia vol. 4 f. $54 \mathrm{r}-55 \mathrm{v}$. Er wird "ministro“ (Okonom) genannt. So wurde auch schon Benedetto Francesi bezeichnet; vgl. CP vol. 137 f. 137r.

119. SC Stamperia Misc. vol. 1 f. 105 r, 114rv.

120. Kopien des Briefes befinden sich in SC Stamperia Misc. vol. $1 \mathrm{f}$. 105r, 115r. Eine Liste der Lettern ist im selben Band f. 110r und in SC Stamperia vol. 4 f. 453r. - M. Galeotti: Della Tipografia Poliglotta, S. 22-23.

121. SC Stamperia Misc. vol. 1 f. 105r, 107rv. SC Stamperia vol. 4 f. 70r, 453r.

122. SC Stamperia vol. 4 f. $67 \mathrm{v}$.

123. SC Stamperia Misc. vol. 1 f. 99r-103r. Er wurde 1751 in Rom geboren. Er war Bibliothekar von Kardinal L. Antonelli. Starb 1826. Seine Bibliographie befindet sich in: G. Amati: Bibliografia Romana, Roma 1880, 48-66.

124. SC Stamperia vol. 4 f. 200 .

125. SC Stamperia vol. 4 f. 213 r.

126. SC Stamperia vol. 4 f. 254 r. 
127. SC Stamperia vol. 4 f. $411 \mathrm{v}$.

128. Diario. Acta vol. 175 f. $43 \mathrm{r}$.

129. SC Stamperia vol. 4 f. 435v. G. Moroni schätzt den Wert auf 170.000 Scudi, in: Dizionario di erudizione storico-ecclesiastica vol. LXIX, Venezia 1854, 238.

130. SC Stamperia vol. 4 f. 519v, 435r.

131. SC Stamperia Misc. vol. 1 f. 162r. Schon am 8. August 1813 wurde ein Gesuch gestelle, die Druckerei wieder zu eröffnen. Aber Paris antwortete nicht; vgl. Diario. Acta vol. 175 f. $138 \mathrm{v}$.

132. Eine Kopie dieses Briefes befindet sich in: SC Stamperia Misc, vol. 1 f. 131r. - Ein weiteres Bittgesuch wurde von Pius VII. gestellt; vgl. A. Huonder: Die Verdienste der katholischen Heidenmission um die Buchdruckerkunst in überseeischen Ländern, Aachen $1923,112$.

133. SC Stamperia vol. 2 f. $170 v$.

134. SC Stamperia vol. 4 f. 450 ; vol. 5 f. 225 r.

135. P. Cullen wurde in Prospect, Irland geboren. Er studierte am Propaganda-Kolleg, an dem er 1829 als Prof. für Exegese ernannt wurde. Gleichzeitig war er auch Direktor der Polyglotta. 1832 wurde er Rektor des irischen Kollegs in Rom. 1850 wurde er zum Erzbisch of von Armagh ernannt, 1866 Kardinal. Er starb 1878 in Dublin.

136. Es gibt mehrere nachgemachte Sammlungen, d. h. sie enthalten Dokumente, die zu verschiedenen Zeiten gedruckt worden sind und die dem internen Gebrauch der Kongregation dienten. Vgl. dazu: J. Dindinger, OMI: Die Sammlungen römischer Missionserlasse. In: „Missionswissenschaft und Religionswissenschaft “ 2: Münster 1939, 125-141. - Bd. 1 wurde 1839, Bd. 2 und Bd. 3 1840, Bd. 4 und Bd. 5 1841, zwei weitere Bände mit einem Anhang und Inhaltsverzeichnissen 1858 veröffentlicht.

137. SC Stamperia vol. 5 f. 33r. Uber seine Verdienste vgl. G. Moroni: Dizionario vol. LXIX, Venezia 1854, 239.

138. SC Stamperia vol. 5 f. $194 \mathrm{v}$.

139. SC Stamperia vol. 6 f. $19 \mathrm{r}$.

140. SC Stamperia vol. 6 f. $19 r$.

141. Antonio Saverio de Luca wurde 1805 in De Bronte, Sizilien, geboren. Er leitete die Polyglotta bis zum Jahre 1845, in dem er zum Bischof von Aversa ernannt wurde. 1863 wurde er Kardinal. Er starb 1883.

142. SC Stamperia vol. 6 f. 588 r.

143. SC Stamperia vol. 5 f. 521r, 535r, 544r, 553r.

144. M. Galeotti: Della Tipografia Poliglotta di Propaglotta di Propaganda Fide, Torino $1866,92$.

145. SC Stamperia vol. 6 f. $603 \mathrm{r}-617 \mathrm{v}$.

146. SC Stamperia vol. 7 f. 621v. Der Titel heißt: Specimen Characterum Typographei S. Concilii Christiano nomine Propagando Sanctissimo Domino Nostro Gregorio XVI Pont. Maximo idem typographeum invisenti oblatum, Romae 1843, $79 \mathrm{~S}$.

147. Vgl. das Gutachten des Advokaten Luigi Antonio de Witten in SC Stamperia vol. 6 f. $28 \mathrm{r}-33 \mathrm{v}$.

148. SC Stamperia vol. 6 f. $341 \mathrm{r}-342 \mathrm{v}$.

149. SC Stamperia vol. 6 f. 430 .

150. SC Stamperia vol. 6 f. $623 \mathrm{r}-629 \mathrm{v}$.

151. SC Stamperia vol. 6 f. 284 r.

152. SC Stamperia vol. 6 f. $214 \mathrm{r}, 284 \mathrm{r}-294 \mathrm{r}$.

153. SC Stamperia vol. 6 f. $274 \mathrm{r}-283 \mathrm{r}, 284 \mathrm{r}-294 \mathrm{r}, 303 \mathrm{r}-310 \mathrm{r}, 363 \mathrm{r}-368 \mathrm{r}$.

154. SC Stamperia vol. 6 f. $177 \mathrm{r}, 469 \mathrm{r}-471 \mathrm{r}$.

155. SC Stamperia vol. 6 f. 349r.

156. SC Stamperia vol. 6 f. 349 r.

157. SC Stamperia vol. 6 f. 399 r-409r.

158. Acta vol. 220 f. $140 \mathrm{Ar}$; SC Stamperia vol. 6 f. 470 , $581 \mathrm{r}$.

159. SC Stamperia vol. 7 f. $12 \mathrm{r}-363 \mathrm{r}$.

160. SC Stamperia vol. 8 f. 232 r.

161. Acta vol. 229,1865, f. $17 \mathrm{r}-25 \mathrm{r}$.

162. Acta vol. 229, 1865, f. 22r-24v. Der lateinische Text und die italienische Ubersetzung befinden sich in: M. Galeotti: Della Tipografia Poliglotta di Propaganda, Torino 1866, 98-100.

163. M. Galeotti, a. a. O. 35-37. 
164. Die Ausgabe bereitete Giovanni Bollig vor, der Professor für Orientalische Sprachen am Collegio Romano und an der Sapienza-Universität war.

165. SC Stamperia vol. 7 f. 495 r.

166. Anno primo 1865-1866. Der erste Herausgeber war Pietro Avanzini. 1870 wurde der Titel geändert in „Acta Sanctae Sedis in compendium opportune redacta et illustrata“. Sie veröffentlichten die wichtigsten Dekrete des Papstes und der römischen Kurie. Am 23. März 1904 wurde die Zeitschrift als, authentisches und offizielles“ Organ für die Akten des Heiligen Stuhls erklärt. Vgl. „Acta Sanctae Sedis" vol. 37, 1904/1905, 4.

167. SC Stamperia vol. 7 f. 507r. Bd. I erschien 1869; II: 1870; III: 1871; IV: 1872; V (das N. T.): 1868. Bd. I-II wurden von Carlo Vercellone und Gaetano Sergio; Bd. III-V von Carlo Vercellone und Giuseppe Cozza bearbeitet. Vgl. S. Garofalo: Nuove note sulla edizione in Fac-simile del Codex Vaticanus (B). In: Mélanges Eugène Tisserant vol. I (Studi e Testi Nr. 231), Città del Vaticano 1964, 217-227.

168. SC Stamperia vol. 7 f. $676 \mathrm{r}-678 \mathrm{r}$.

169. Pallottini sollte die Sammlung „Collectio conclusionum et resolutionum S. Congregationis Concilii Tridentini interpretatum quae prodierunt ab anno 1564 ad annum 1860 per materias digestas" veröffentlichen. Es waren im ganzen 15 Bände geplant; für den Buchstaben A hatte er aber schon zwei Bände vorbereitet. Pallottini klagte, daß der Druck zu langsam vonstatten gehe. Vgl. SC Stamperia vol. 8 f. 148r; Acta vol 237, 1871, f. 105r. - Uber das Verfahren mit Silvetri vgl. SC Stamperia vol. 8 f. 18 r.

170. Die Frage wurde auf friedlichem Wege geregelt; vgl. SC Stamperia vol. $8 \mathrm{f}$. 3r.

171. SC Stamperia vol. 8 f. 61r. Vgl. auch die Bedingungen in Acta vol. 241, 1874, f. 569r bis $576 \mathrm{r}$.

172. SC Stamperia vol. 8 f. 96 r.

173. SC Stamperia vol. 8 f. $426 \mathrm{r}-432 \mathrm{r}$.

174. SC Stamperia vol. 8 f. 452r. - Vgl. auch V. Prinzivalli: Le Missioni Cattoliche al di là dei mari, Roma 1903, 385. Dort werden auch weitere Veröffentlichungen der Polyglotta aus dieser Periode erwähnt, S. 385-390.

175. SC Stamperia vol. 8 f. 465r, 600r, 614r.

176. SC Stamperia vol. 8 f. 661r-663v, 696r.

177. R. de Martinis: Juris Pontificii de Propaganda Fide. Teil I: 6 Bände 1888-1895; Teil II: 1 Bd. 1909. Das Werk wurde von Francesco Rosi-Bernardini begonnen und nach dessen Tod von R. de Martinis fortgesetzt; vgl. Teil I Bd. 1 S. X.

178. Vgl. W. Henkel: The Polyglot Printing-office during the 18th and 19th century. In: J. Metzler: Sacrae Congregationis de Propaganda Fide Memoria Rerum vol. II, 1973, 313.

179. W. Henkel: a. a. O., 314.

180. Der vollständige Titel lautet: Collectanea S. C. de Propaganda Fide. Seu Decreta, Instructiones, Rescripta pro Apostolicis missionaribus ex tabulario eiusdem S. Congregationis deprompta, Roma 1893. 1 Bd. - Die Ausgabe von 1907 wurde in zwei Bänden veröffentlicht.

181. Vgl. H. Mathes: Tipografia Poliglotta Vaticana. In: „Der Polygraph“, Frankfurt 1971,661 .

182. Vgl. dazu R. Prélot: La presse Catholique dans le Tiers Monde, Paris 1968, 191-222.

183. Die Entscheidung wurde von Papst Pius X. am 5. November 1908 approbiert. Vgl. W. Henkel: a. a. O., 314. N. Vian: Tipografia Vaticana. In: Enciclopedia Cattolica XII col. 1135-1137. Ein Teil der Druckerei wurde an die Società Tip. Ed. Romana verkauft.

\section{S U M M A R Y}

The author, chief librarian at the Propaganda library in Rome gives the exact and detailed history of the Propaganda printing press from its beginning in 1622, till its merger with the Vatican printing press in 1908 . The description is based on the respective documents from the Propaganda archives. The foundation, development, and the critical moments are 
described, as well as the publications up till 1700, the new rise of the Polyglotta under Ruggieri and Amaduzzi. The developments during the time of the French revolution, the new beginnings and modernization in the almost 300 years of the existence of the Propaganda press are presented. The difficult economical situation in the second half of the 19th century finally, and simultaneously with the new organization of the Vatican curia, led towards the merger of the Propaganda Fide with the Vatican printing press into the present Tipografia Poliglotta Vaticana.

\section{RESUME}

L'auteur traduit de façon objective et exacte l'histoire de l'imprimerie de la Propaganda Fide au service de la propagation de la foi de ses débuts en 1622 jusqu'à sa fusion avec l'imprimerie du Vatican, en 1908, en „Tipografia Poliglotta Vaticana“; il justifie continuellement sa description à l'aide de documents s'y rapportant. Fondation, agrandissement et temps de crise, publication jusqu'en 1700, nouvel essor de la Poliglotta sous Ruggeri et Amaduzzi, l'époque de la Révolution française, nouveau début et modernisation, l'histoire, vieillle de près de trois siècles, avec ses hauts et ses bas, renaît sous les yeux du lecteur. La situation économique peu favorable de la deuxième moitié du $19 \mathrm{e}$ siècle aboutit finalement entre autre, à l'occasion de la réorganisation de la Curie Romaine, à la fusion de l'imprimerie de la Propaganda Fide avec celle du Vatican pour donner l'actuelle "Tipografia Poliglotta Vaticana ${ }^{\alpha}$.

\section{RE S U M E N}

El autor reproduce objetiva y detalladamente, con amplia documentación, la historia de la imprenta de la Propaganda Fide al servicio de la propagación de la fe desde sus comienzos en 1622 hasta su unión con la Imprenta Vaticana en 1908, fusión de la que nació la "Tipografia Poliglotta Vaticana". Ante el lector aparecen la fundación, desarrollo y épocas de crisis, publicaciones hasta 1700 , nueva pujanza bajo Ruggieri y Amaduzzi, la época de la Revolución Francesa, nuevo comienzo y modernización, en suma casi tres siglos de historia, con todos sus altibajos. La desfavorable situación económica en la segunda mitad del siglo XIX condujo por último, a raiz de la reestructuración de la Curia romana, a la fusión de la imprenta de la Propaganda Fide con la Vaticana para formar la actual "Tipografia Poliglotta Vaticana". 